\title{
Internship Effect Prediction for Physical Education Majors Based on Artificial Neural Network
}

\author{
https://doi.org/10.3991/ijet.v16i24.27839 \\ Bo Yang \\ Heilongjiang Bayi Agricultural University, Daqing, China \\ mfh001@byau.edu.cn
}

\begin{abstract}
Professional internship offers college students a golden chance to apply their theoretical knowledge to practice. Through internship, physical education (PE) majors can match the professional knowledge and skills learned at school with the competencies required by actual jobs. The relevant studies at home and abroad mainly attempt to improve the internship effect. This paper explores the influence of the diversity of job competencies on the internship effect of PE majors, and establishes a prediction model based on artificial neural network (ANN). Firstly, an evaluation index system (EIS) was constructed for the internship quality of PE majors, and a table was prepared for four types of internship jobs for PE majors, as well as their core competences. Then, the sample data for quality evaluation of PE majors' internship were preprocessed and subjected to feature extraction, in the light of their sequential property. After that, a prediction model was proposed for the internship quality of PE majors, along with its optimization algorithm. The proposed model was proved effective through experiments.
\end{abstract}

Keywords - internship of physical education (PE) majors, internship effect, diversity of job competences, artificial neural network (ANN)

\section{Introduction}

Professional internship is the most common way for college students to gain professional experience, and attracts much attention from institutions of higher learning across the globe. It is widely recognized as a golden chance for college students to apply their theoretical knowledge to practice [1-6]. As a link of talent cultivation in college, professional internship aims to improve the students' mastery of professional knowledge by combining learning with working, and enhance their adaptability to jobs and professional literacy [7-14]. Through professional internship, physical education (PE) majors can fully experience the actual jobs on PE education, sports product marketing, and sports events planning, and match the professional knowledge and skills learned at school with the competencies required by actual jobs. The colleges can rely on professional internship to fulfil their task of cultivating high-quality PE talents, and serving the society with PE. 
The teaching effect of professional internship bears on the employment of graduates, and determines how well the students adapt to the needs of future careers. Zhou and Deng [15] analyzed the contents and significance of professional internship, demonstrated the necessity of effective PE teaching in higher vocational colleges, and probed deep into the effectiveness of PE teaching in these colleges. On this basis, concrete measures were presented to enhance the effectiveness of PE teaching in terms of contents and significance. Gao and $\mathrm{Xu}$ [16] explored the construction of PE infrastructure from multiple aspects: deepening the cognition of education practice, strengthening ideological construction, establishing an excellent faculty, improving the integrated quality of interns, supporting the construction of sports facilities and practice bases, and perfecting the rules and regulations on teaching and internship. Cui and Wang [17] suggested that PE internship and practice courses can effectively make up for the defects of higher education, and recommended to survey the material culture and psychological culture of students through PE internship and practice courses, thereby improving their interest, attitude, and awareness of physical exercise.

The existing studies at home and abroad mainly try to improve the implementation models of internship, adjust the time distribution of internship in the learning cycle, and find other ways to enhance the internship effect. Some scholars have attempted to manage internship with the latest information technology (IT) and Internet technology, hoping to improve the students' internship effect. This paper explores the action and effect of the diversity of job competencies on the internship effect of PE majors, and predicts the PE majors' internship effect under the diversity of job competencies. The main contents are as follows: (1) Setting up an evaluation index system (EIS) for the internship quality of PE majors, and drawing a table for four types of internship jobs for PE majors, as well as their core competences; (2) Conducting processing and feature extraction of the sample data for quality evaluation of PE majors' internship, in the light of their sequential property; (3) Proposing a prediction model for the internship quality of PE majors, along with its optimization algorithm, and verifying the model through experiments.

\section{EIS construction}

The PE major requires its students to acquire a strong practical ability. One of the most important practical teaching links is professional internship. The training quality of PE professionals is greatly affected by the diversity of competences required by the internship jobs. During the internship, the students can gradually understand the competences required by actual jobs. To meet the corporate demand, the students must acquire diverse job competencies. Table 1 lists four types of internship jobs for PE majors, and their core competencies. 
Table 1. Four types of internship jobs and their core competencies

\begin{tabular}{|c|c|c|}
\hline Type & Functions & Core competencies \\
\hline $\begin{array}{l}\text { PE teaching } \\
\text { (College teachers, pri- } \\
\text { mary and secondary } \\
\text { school teachers, training } \\
\text { institutions teachers) }\end{array}$ & $\begin{array}{l}\text { 1. Impart PE knowledge and skills to students; } \\
\text { 2. Cultivate correct PE view for students; } \\
\text { 3. Train physical practice ability of students. }\end{array}$ & $\begin{array}{l}\text { 1. Teaching ability } \\
\text { 2. Teaching re- } \\
\text { search ability }\end{array}$ \\
\hline $\begin{array}{l}\text { Sports product/service } \\
\text { marketing } \\
\text { (Product/service sales di- } \\
\text { rector and assistant, } \\
\text { sports product/service } \\
\text { shopping guide) }\end{array}$ & $\begin{array}{l}\text { 1. Complete market survey and analysis of sports prod- } \\
\text { ucts/services; } \\
\text { 2. Develop marketing plans for sports products/services; } \\
\text { 3. Implement flexible sales of sports products/services; } \\
\text { 4. Implement integrated management of various sports } \\
\text { product/service marketing programs. }\end{array}$ & $\begin{array}{l}\text { 1. Marketing abil- } \\
\text { ity } \\
\text { 2. Marketing man- } \\
\text { agement ability }\end{array}$ \\
\hline $\begin{array}{l}\text { Sports event planning } \\
\text { (Execution specialist, } \\
\text { planning specialist, plan- } \\
\text { ning director and assis- } \\
\text { tant) }\end{array}$ & $\begin{array}{l}\text { 1. Prepare event plans; } \\
\text { 2. Develop event rules; } \\
\text { 3. Invite sponsors; } \\
\text { 4. Expand the influence of events, and produce multi-fac- } \\
\text { eted social benefits; } \\
\text { 5. Maintain normal operation of events; } \\
\text { 6. Evaluate results of events. }\end{array}$ & $\begin{array}{l}\text { 1. Event planning } \\
\text { ability } \\
\text { 2. Event operation } \\
\text { ability }\end{array}$ \\
\hline $\begin{array}{l}\text { Sports venue } \\
\text { management } \\
\text { (Site workers, venue op- } \\
\text { eration director, venue } \\
\text { director and assistant) }\end{array}$ & $\begin{array}{l}\text { 1. Coordinate and manage venue personnel; } \\
\text { 2. Implement financial operation and property manage- } \\
\text { ment of venues; } \\
\text { 3. Check and regularly maintain venue facilities and } \\
\text { equipment; } \\
\text { 4. Maintain normal operation of venues. }\end{array}$ & $\begin{array}{l}\text { 1. Venue operation } \\
\text { 2. Venue manage- } \\
\text { ment }\end{array}$ \\
\hline
\end{tabular}

As shown in Table 1, the professional internship requires the PE majors to have four types of abilities: basic personal abilities, job cognition abilities, job qualities, and job extension abilities. Referring to books on education internship, education evaluation, PE internship instructions, this paper finalizes an EIS of six core indices, namely, internship preparation A1, internship ability A2, internship effect A3, special PE instruction ability A4, internship file writing ability A5, and internship performance A6, through repeated discussions with advisers and experts of PE internship. Figure 1 shows the construction process of the EIS.

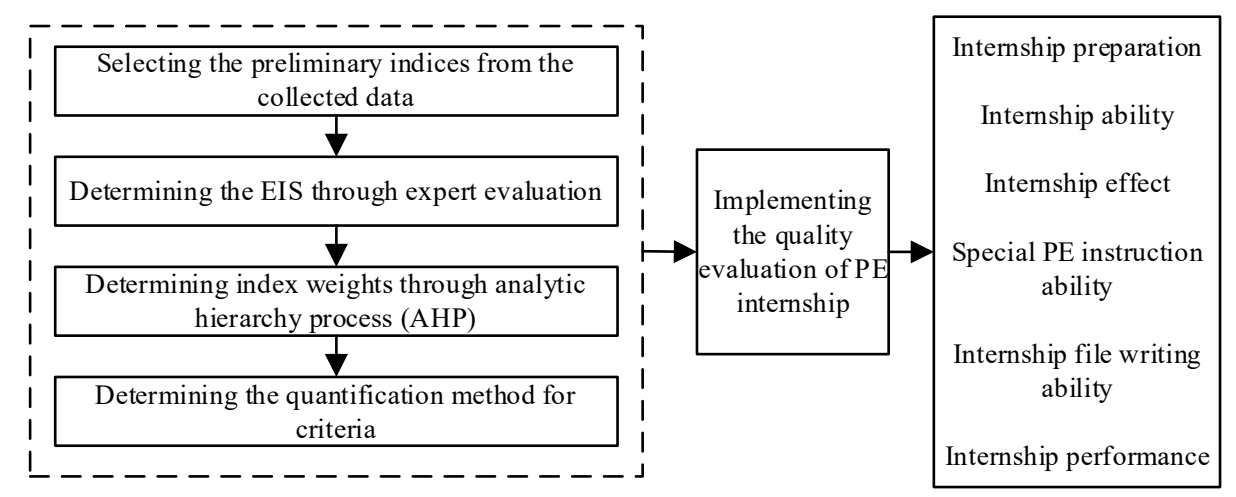

Fig. 1. EIS construction 


\section{Construction of prediction model}

\subsection{Model optimization algorithm}

Deep learning (DL) network has a more complicated structure, and more hidden layers than normal neural networks. As a result, it is more difficult for a DL network to converge to the optimal solution than a normal neural network. In general, ANNs can be optimized by two kinds of algorithms: first-order optimization algorithms like gradient descent (GD) and momentum technology, and second-order optimization algorithms like Mersenne Twister (MT) algorithm. Owing to the excessive cost of the MT algorithm, this paper chooses to improve one of the first-order optimization algorithms, i.e., standard GD, to optimize the ANN. Let $\operatorname{LOSS}(\omega)$ be the loss function containing all parameters, and $\beta$ be the learning rate. The standard GD method can be expressed as:

$$
\omega_{i}=\omega_{i}-\beta \frac{\partial}{\partial \omega_{i}} \operatorname{LOSS}(\omega)
$$

The standard GD faces a series of problems, such as poor effect on massive data, proneness to the local minimum trap, and redundancy of weight updates. To overcome these problems, the momentum technology and adaptive method were introduced to the stochastic GD (SGD). The specific ideas are as follows: (1) finding the optimal solution; (2) minimizing the loss function; (3) updating model parameters; (4) implementing a new round of iteration; (5) enhancing the momentum in relevant directions and weakening that in unrelated directions based on the momentum technology; (6) iteratively completing model convergence. The improved method can speed up the training of standard GD. Let $\delta$ be the momentum component, i.e., the component of the parameters updated in the previous moment. Then, the standard GD can be improved by introducing $\delta$ to update the parameters at the current moment:

$$
\begin{gathered}
U(q)=\delta U(q-1)+\beta \frac{\partial \operatorname{LosS}(\omega)}{\partial \omega_{i}} \\
\omega_{i}=\omega_{i}-U(q)
\end{gathered}
$$

where, $\delta$ is generally set to 0.9 . Another way to optimize the standard GD is to replace $\omega$ with $\omega-\delta U(q-1)$ in the updating formula. After the replacement, the improved GD is more sensitive to parameter update, and capable of predicting the research problem, to a certain extent.

To help our ANN adapt to different parameters and achieve fast learning, the historical gradients of all network parameters at each moment could be calculated, and adaptive learning rates be set for different network parameters. Let $h_{q, i}$ be the gradient of the loss function of parameter $\omega_{i}$ at time q; $H_{q, i i}$ be the quadratic sum of the gradients of all parameters prior to time $\mathrm{q} ; \beta$ be the global learning rate. Then, we have:

$$
\omega_{q+1, i}=\omega_{q, i}-\frac{\beta}{\sqrt{H_{q, i i}+\tau}} \cdot h_{q, i}
$$


On this basis, the momentum change of each parameter can be calculated by timeadaptive method. Let $h_{q, i}$ be the gradient of the loss function for parameter $\omega_{i}$ at time q. Then, the revised first-order estimation equation with gradient can be expressed as:

$$
\hat{n}_{q, i}=\frac{\alpha_{1} \cdot n_{q-1, i}+\left(1-\alpha_{1}\right) \cdot h_{q, i}}{1-\alpha_{2}^{q}}
$$

The revised second-order estimation equation can be expressed as:

$$
\hat{u}_{q, i}=\frac{\alpha_{2} \cdot u_{q-1, i}+\left(1-\alpha_{2}\right) \cdot h_{q, i}^{2}}{1-\alpha_{2}^{q}}
$$

The final improved GD can be expressed as:

$$
\omega_{q, i}=\omega_{q-1, i}-\beta \cdot \frac{\hat{n}_{q}}{\sqrt{\hat{u}_{q}+\tau}}
$$

\subsection{Construction of prediction model}

This paper adopts the decomposition-integration framework (Figure 2) to predict the quality index of the professional internship of PE majors. The framework mainly involves four steps: data preprocessing, signal decomposition, independent prediction, and integrated prediction. To improve the stability and accuracy of network prediction, this paper extends the framework into the improved long short-term memory (LSTM) network with elastic net penalty for the forecast of the quality index of the professional internship of PE majors, carries out variational mode decomposition (VMD) on key sample data under decomposition-integration paradigm, and optimizes the parameter estimation and other steps of the network model by a network search algorithm.

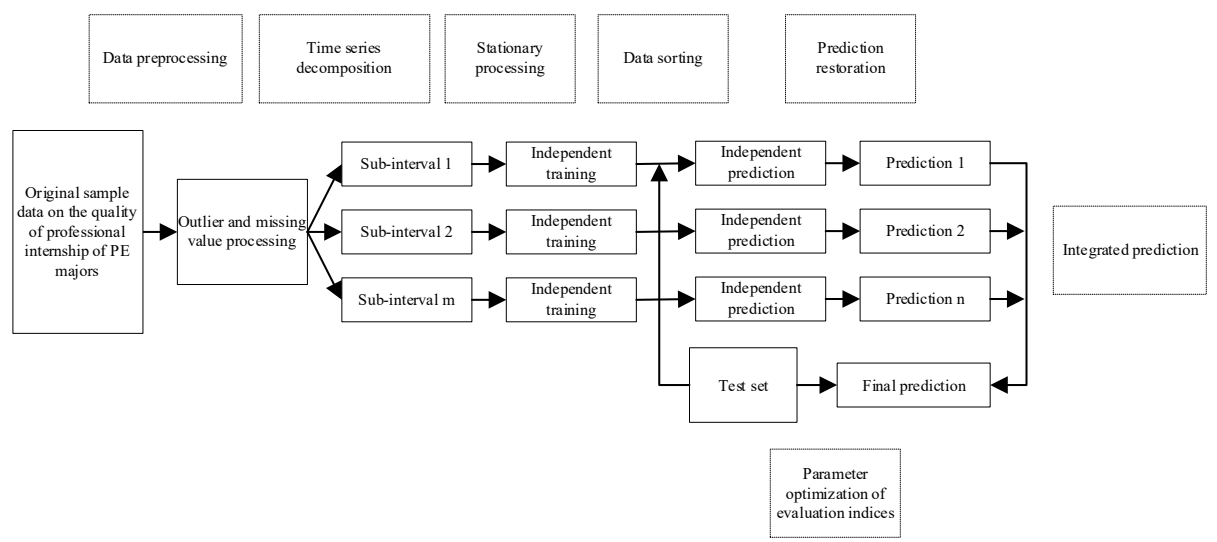

Fig. 2. Flow of quality prediction of professional internship of PE majors

The VMD decompose the time series on the professional internship quality of PE majors into L intrinsic mode functions (IMFs). Unlike traditional empirical mode decomposition (EMD), VMD adopts a strong constraint that minimizes the sum of the 
bandwidths of L IMFs, i.e., the target signal is set as the combination of sub-time series with the minimum sum of bandwidths. All such combinations are obtained through iteration. Therefore, the instantaneous spectrum of the combination of sub-time series in VMD is physically meaningful. The VMD can be implemented in the following steps:

- Step 1. Spectrum acquisition

Obtain the one-sided spectrum of each intrinsic mode $v_{l}$ through Fourier transform:

$$
\left(\gamma(q)+\frac{j}{\pi q}\right) \times v_{l}(q)
$$

- Step 2. Spectrum shift

Shift the spectrum of each intrinsic mode $v_{l}$ to the baseband:

$$
\left(\gamma(q)+\frac{j}{\pi q}\right) \times e^{-j t_{l} q}
$$

- Step 3. Problem solving

Let $\left\{v_{k}\right\}=\left\{v_{1}, v_{2}, \ldots, v_{l}\right\}$ be all IMFs; $\left\{t_{k}\right\}=\left\{t_{1}, t_{2}, \ldots, t_{l}\right\}$ be the central frequencies of the modes; $\gamma(q)$ be the Dirichlet function; * be the convolutional operator; $r$ be the original time series of the quality data on professional internship of PE majors. The decomposition process was realized by minimizing the square of the $\mathrm{L}_{2}$ norm:

$$
\min _{\left\{v_{l}\right\}\left\{t_{l}\right\}}\left\{\sum_{l}\left\|\partial_{q}\left[\left(\gamma(q)+\frac{j}{\pi q}\right) * v_{l}(q)\right] e^{-j t_{l} q}\right\|_{2}^{2}\right\}
$$

The LSTM network introduces the state parameters and three gates (input gate, forget gate, and output gate) to every node in the recurrent neural network (RNN) (Figure 3 ). Whether the propagation is forward or backward, the outputs of the input gate, forget gate, and output gate of LSTM are influenced by the same factors: data samples of the input layer, peephole information, and hidden layer information; the state parameters are affected by two issues: the output of the input gate, and the hidden layer information. The structures of the three gates jointly determine the state $d_{q}$ of node and state $f_{q}$ of hidden layer in the neural network at the current moment q. The general computing process of the LSTM network is briefly introduced as follows:

The network model employs the sigmoid function, which converts variables into [0, 1], and the hyperbolic tangent tanh function, which converts variables into $[-1,1]$. The Hadamard multiplier of the matrix is denoted as $\odot$, the input at time q as $a_{q}$, the hidden layer state at time $\mathrm{q}$ as $f_{q}$, weight as $\mathrm{T}$, and bias as $\mathrm{p}$. Then, we have:

$$
s_{q}=\operatorname{sigmoid}\left(T_{i} \cdot\left[f_{q-1}, a_{q}\right]+p_{i}\right)
$$




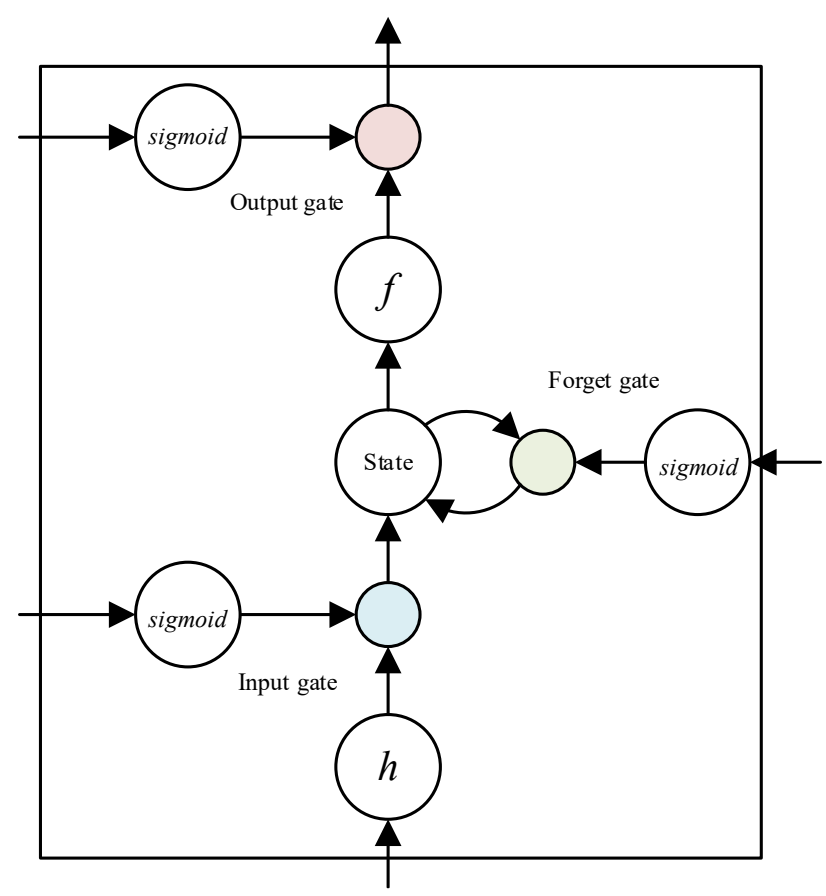

Fig. 3. Structure of LSTM unit

Intuitively, $s_{q}$ can be viewed as a function that determines the amount of new information to be stored in the node at the current moment. Then, $g_{q}$ can be considered as a function about the amount of information forgotten by the node at the previous moment:

$$
\begin{gathered}
g_{q}=\operatorname{sigmoid}\left(T_{g} \cdot\left[f_{q-1}, a_{q}\right]+p_{g}\right) \\
h_{q}=\tanh \left(T_{h} \cdot\left[f_{q-1}, a_{q}\right]+p_{h}\right)
\end{gathered}
$$

Similarly, $e_{q}$ can be viewed as a function about the amount of output information:

$$
e_{q}=\operatorname{sigmoid}\left(T_{e} \cdot\left[f_{q-1}, a_{q}\right]+p_{e}\right)
$$

At the current moment q, the state $d_{q}$ of neural network node can be expressed as:

$$
d_{q}=g_{q} \odot d_{q-1}+s_{q} \odot h_{q}
$$

The hidden layer state $f_{q}$ can be expressed as:

$$
f_{q}=e_{q} \odot \tanh \left(d_{q}\right)
$$

The above analysis shows that $g_{q}, s_{q}$ and $e_{q}$ jointly determines the update of the state $d_{q}$ of the node and the state $f_{q}$ of hidden layer at time q.

To prevent overfitting in model learning, the regularization term needs to be added to the objective function as a penalty term. In statistical analysis, the three common 
regularization terms are Lasso regularization, ridge regression regularization, and elastic net regularization. For a regression model, Lasso regularization, and ridge regression regularization respectively introduce the first- and second-order norms of the coefficient matrix to the objective function to be optimized. Meanwhile, the elastic net regularization introduces the compromised form of these two regularization terms.

In our prediction model, three weights need to be learned, including partial connection weight, input connection weight, and recursive connection weight. To enhance the generalization ability of the prediction model, this paper sets different elastic net regularization terms for the learning of the three weights. The penalty term of the input weight can be expressed as:

$$
\min _{\theta}\left\{\frac{1}{Q} \sum_{q=1}^{Q} \sum_{i=1}^{m}\left(b_{q i}-\bar{b}_{q i}\right)^{2}+\mu_{1}\|\theta\|_{1}+\mu_{2}\|\theta\|_{2}^{2}\right\}
$$

If $\mu 1=0$ and $\mu 2 \neq 0$, formula (28) can be viewed as the penalty of ridge regression regularization; if $\mu 1 \neq 0$ and $\mu 2=0$, formula (28) can be viewed as the penalty of Lasso regression; if $\mu 1 \neq 0$ and $\mu 2 \neq 0$, formula (28) can be viewed as the penalty of elastic net regression; if $\mu 1=\mu 2=0$, no penalty is imposed on the input weight.

\section{$4 \quad$ Experiments and results analysis}

The above analysis results were used to verify the proposed EIS for the internship quality of PE majors, which considers the diversity of job competencies. The judgement matrix of the core indices was established through pairwise comparison and index rating (Table 2). Based on the judgement matrix, the weights of the core indices were determined as $0.241,0.317,0.154,0.122,0.074$, and 0.092 . It can be inferred that the internship ability, which contains PE knowledge and skills, had the highest weight, while the internship file writing ability had the smallest weight. Hence, PE majors should complete internship according to the importance reflected by the weights of the core indices, in the light of their own conditions.

Table 2. Judgement matrix of primary indices

\begin{tabular}{|l|c|c|c|c|c|c|}
\hline \multicolumn{1}{|c|}{ Index number } & A1 & A2 & A3 & A4 & A5 & A6 \\
\hline A1 & 1 & 2 & 3 & 4 & 5 & 6 \\
\hline A2 & $1 / 2$ & 1 & 2 & 3 & 4 & 5 \\
\hline A3 & $1 / 3$ & $1 / 2$ & 1 & 2 & 3 & 4 \\
\hline A4 & $1 / 4$ & $1 / 3$ & $1 / 2$ & 1 & 2 & 3 \\
\hline A5 & $1 / 5$ & $1 / 4$ & $1 / 3$ & $1 / 2$ & 1 & 2 \\
\hline A6 & $1 / 6$ & $1 / 5$ & $1 / 4$ & $1 / 3$ & $1 / 2$ & 1 \\
\hline
\end{tabular}

To verify its effectiveness, the proposed prediction model for the professional internship quality of PE majors was compared with several models: the model without VMD (model 2), the model without network search algorithm (model 3), multistep LSTM prediction model (model 4), traditional LSTM model (model 5), and backpropagation (BP) neural network model (model 6). The prediction performance of the latter 
three models depends directly on model parameters and hyperparameters. Therefore, the parameters and hyperparameters of model 4 were configured the same as those of model 2, all of which were optimized through network search. The other models were not optimized through network search. As for the optimal parameters of the LSTM, the number of nodes, the maximum number of iterations, batch size, and time step length were set to $50,100,100$, and 1 , in turn. The optimization coefficient of the elastic net penalty was fixed at 0.005 . Table 3 lists the empirical results on the predicted quality of PE professional internship by different models. Figure 4 shows the corresponding prediction effects.

Table 3. Errors of different prediction models

\begin{tabular}{|l|c|c|}
\hline \multirow{2}{*}{ Model number } & \multicolumn{2}{|c|}{ Errors } \\
\cline { 2 - 3 } & $\boldsymbol{R M S E}$ & $\boldsymbol{M A E}$ \\
\hline 1 & 24.532 & 21.816 \\
\hline 2 & 41.681 & 27.549 \\
\hline 3 & 46.956 & 27.651 \\
\hline 4 & 57.273 & 32.756 \\
\hline 5 & 61.354 & 33.295 \\
\hline 6 & 64.758 & 37.678 \\
\hline
\end{tabular}

Note: RMSE and MAE are short for root mean square error and mean absolute error, respectively.

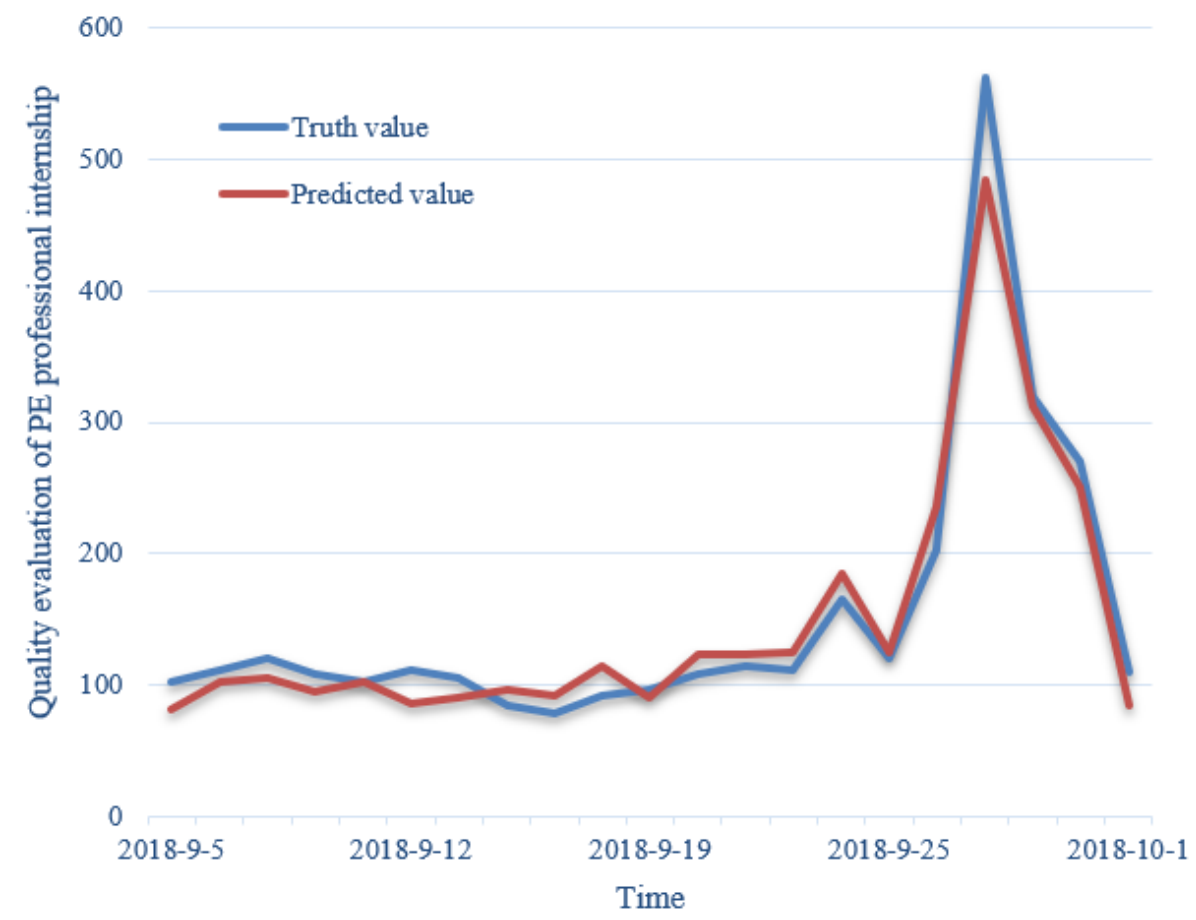

Fig. 4. Prediction effects 
The RMSEs and MAEs in Table 3 indicate that our prediction model performed better than the other models, because its RMSE and MAE reductions were more significant than those of any other model (Table 4).

Table 4. Error reductions of other models

\begin{tabular}{|l|c|c|c|c|c|c|}
\hline \multirow{2}{*}{} & \multirow{2}{*}{ Metrics } & \multicolumn{5}{|c|}{ Model number } \\
\cline { 3 - 7 } & & $\mathbf{2}$ & $\mathbf{3}$ & $\mathbf{4}$ & $\mathbf{5}$ & $\mathbf{6}$ \\
\hline \multirow{2}{*}{ Errors } & EMSE & 37.512 & 47.651 & 58.623 & 87.604 & 62.763 \\
\cline { 2 - 7 } & MAE & 17.635 & 18.452 & 34.512 & 36.543 & 43.531 \\
\hline
\end{tabular}

The superiority of our model comes from four factors: (1) the decomposition-integration framework can effectively overcome the non-stationarity of the time series on professional PE internship quality; (2) the VMD is much more flexible than the conventional EMD, making the prediction more realistic and accurate; (3) the regularization penalty of the neural network prevents overfitting, and enhances prediction stability; (4) the network search algorithm effectively optimizes the proposed neural network.

\section{Conclusions}

This paper develops an ANN prediction model for the internship effect of PE majors in the context of the diversity of job competencies. Firstly, the authors summarized four types of jobs in professional PE internship, analyzed their core competencies, and set up an EIS for the quality of the professional internship of PE majors. Afterwards, the sequential features of the raw data on the internship quality of PE majors were analyzed, and the strategies were presented for sample preprocessing and feature selection. Next, a quality prediction model was established for professional PE internship, and verified through experiments. Based on the experimental results, the authors demonstrated the prediction effect of our model on the quality of professional PE internship. The RMSEs and MAEs of various models show that our prediction model is superior than all contrastive methods.

\section{References}

[1] Wambeke, B. (2020). Building Bridges-Spanning the gap between the classroom and professional practice. In 2020 ASEE Virtual Annual Conference Content Access. https://doi.org/10.18260/1-2--34236

[2] Chowdhury, T., Perry, L., Murzi, H., Vicente, S. (2020). Professional practice in engineering education: lessons learned from students participating in internships. SEFI 48th Annual Conference Engaging Engineering Education, Proceedings, 148-157.

[3] Hrmo, R., Krištofiaková, L., Miština, J. (2019). Emotional Intelligence of Secondary Technical School Students and Its Impact on Their Employment in Professional Practice. In International Conference on Interactive Collaborative Learning, 824-834. https://doi.org/10. 1007/978-3-030-40271-6_81 
[4] Xia, Y., Cutler, S., McFadden, D. (2020). Collaborative Project-based Learning Approach to the Enculturation of Senior Engineering Students into the Professional Engineering Practice of Teamwork. In 2020 ASEE Virtual Annual Conference Content Access. https://doi.org/10.18260/1-2--34299

[5] Zhang, Q.S., Yang, D., Fang, P.J., Liu, N.N., Zhang, L. (2020). Develop Academic Question Recommender Based on Bayesian Network for Personalizing Student's Practice, International Journal of Emerging Technologies in Learning, 15(8): 4-19. https://doi.org/10.3991/ ijet.v15i18.11594

[6] Chumchuen, N., Klinbumrung, K., Meesomklin, S. (2020). Professional Teaching Practice Through MIAP based Integrated Learning Activities for Electrical Engineering Education. In 2020 5th International STEM Education Conference (iSTEM-Ed), 95-98. https://doi.org/10.1109/istem-ed50324.2020.9332796

[7] Wang, X.L., Zhang, R.X., Li, Z.X., Chen, J.L. (2020). Analysis on Management of Job Burnout of Counselors in Chinese Colleges Based on Game Theory, International Journal of Emerging Technologies in Learning, 15(17): 207-220. https://doi.org/10.3991/ ijet.v15i17.16739

[8] Liu, D.D., Li, R.W., Sun, Z.F., Liu, J.A., Chen, C. (2010). The application of Virtual Reality in the practice course of physical education. In 2010 4th International Conference on Distance Learning and Education, 78-80. https://doi.org/10.1109/icdle.2010.5606035

[9] Fang, J., Wu, Y., Yan, R., Zhang, J. (2008). Practice and Research on Credit System Administration in College Physical Education. In 2008 IEEE International Symposium on Knowledge Acquisition and Modeling Workshop, 899-902. https://doi.org/10.1109/ kamw.2008.4810637

[10] Zhu, J. (2021). Factors Affecting the Physical Education Practice Teaching Effect of University Students. In 2021 2nd International Conference on Computers, Information Processing and Advanced Education, 202-208. https://doi.org/10.1145/3456887.3456932

[11] Zhang, Y. (2021). Evaluation System of College Physical Education Practice Teaching Based on Big Data. In Journal of Physics: Conference Series, 1852(2): 022012. https://doi.org/10.1088/1742-6596/1852/2/022012

[12] Yang, Z. (2021). Advantages of Computer Information Technology in Physical Education Teaching Practice. In The International Conference on Cyber Security Intelligence and Analytics, 1343: 688-695. https://doi.org/10.1007/978-3-030-69999-4 94

[13] Ren, X. (2021). The Practice and Thinking of Computer Virtual Reality Technology in the Teaching Mode of Physical Education in Colleges and Universities. In Journal of Physics: Conference Series, 1744(4): 042020. https://doi.org/10.1088/1742-6596/1744/4/042020

[14] Li, W. (2020). Research on the Practice Path of Integrating Physical Education Classroom Teaching into Ideological and Political Education Based on Computer Analysis. In Journal of Physics: Conference Series, 1648(3): 032082. https://doi.org/10.1088/1742-6596/ $\underline{1648 / 3 / 032082}$

[15] Zhou, R., Deng, Y. (2014). Study on the effectiveness of physical education practice courses in higher vocational schools. Journal of Chemical and Pharmaceutical Research, 6(7): 917 921.

[16] Gao, Z., Xu, Q. (2014). Study on the Strategy for the Construction of Physical Education Practice Base. 2014 International Conference on Mechatronics, Electronic, Industrial and Control Engineering, MEIC 2014, 1518-1521. https://doi.org/10.2991/meic-14.2014.336

[17] Cui, R., Wang, Y. (2012). Research on Teaching Practice of Hidden Curriculum in University Physical Education. In Future Computer, Communication, Control and Automation, 119: 357-362. https://doi.org/10.1007/978-3-642-25538-0 52 


\section{$7 \quad$ Author}

Bo Yang, graduated from Chengdu Sport University with a master's degree, majoring in physical education and training. Yang Bo is now a teacher and lecturer in the Sports Teaching and Research Department of Heilongjiang Bayi Agricultural University, mainly engaged in the teaching of university PE courses, and concurrently serving as the head coach of the swimming team of Heilongjiang Bayi Agricultural University. During the 2008-2020 period as the head coach, the team members had won 2 gold medals, 1 silver medal and 4 bronze medals in the University Games of Heilongjiang Province. Yang Bo had published 12 provincial-level papers and 1 monograph, and had 1 patent for utility models (email: mfh001@byau.edu.cn).

Article submitted 2021-10-06. Resubmitted 2021-10-30. Final acceptance 2021-10-30. Final version published as submitted by the author. 